\title{
Electrodeposition Process for the Fabrication of Copper Dendrites Film with Stable Superhydrophobicity
}

\author{
Huan $S_{E N N^{1)}}$, Fengping $W A N G^{1 \dagger \dagger}$, Yanwei $D I N G^{1)}$ and Jun YANG ${ }^{2)}$ \\ 1) Faculty of Chemistry and Chemical Engineering, Liaoning Normal University, Dalian 116029, China \\ 2) School of Materials Science and Engineering, Dalian Jiaotong University, Dalian 116028, China \\ [Manuscript received 17 April 2013, in revised form 19 August 2013] \\ (c) The Chinese Society for Metals and Springer-Verlag Berlin Heidelberg
}

\begin{abstract}
Copper dendrites on the copper surface were successfully prepared by electrodeposition in acidic copper sulfate aqueous solution containing ethanol. The XRD (X-ray diffraction) patterns indicate that the $\mathrm{Cu}$ dendrites possess fcc (face-centered cubic) crystal structure. The contact angle of the as-prepared $\mathrm{Cu}$ dendrites surface without palmitic acid modification is almost $0^{\circ}$ and the surface is completely wetted by water. After modified with palmitic acid, the $\mathrm{Cu}$ dendrite surface shows superhydrophobicity with a contact angle of $160^{\circ}$. The polarization curves reveal that the superhydrophobic $\mathrm{Cu}$ dendrites surface exhibits a distinct passivation phenomenon, which could provide enhanced corrosion resistance for the substrate in the aqueous solution. The weight loss measurements show that the corrosion values of superhydrophobic surface is much lower than that of the bare copper, further meaning that the as-prepared surface has the anticorrosion performance.
\end{abstract}

\section{KEY WORDS: Copper; Electrodeposition; Superhydrophobic surface; Ethanol}

\section{Introduction}

There has been intense interest in nanostructures with different shapes and sizes. Some nanostructures have unique optical, electrical and magnetic properties strongly depending on the morphology ${ }^{[1]}$. Copper and its alloys are among the important engineering metallic materials widely used in electronic industry. Various shapes of copper nanostructures have been synthesized like nanowires, nanoparticles, nanorods and nanotubes ${ }^{[2-4]}$. Metal nanostructures with controlled crystalline orientation, grain size, porosity and crystal morphology are desirable for many applications, including catalysis, microelectronic devices, superhydrophobic surfaces and biological films ${ }^{[5-7]}$. Due to large specific surface area and special structure, copper dendrites have been attracted broad interest. Some of the current methods available to generate superhydrophobic surface in laboratory using a wide variety of techniques include colloidal chem-

† Corresponding author. Prof., Ph.D.; Tel.: 13804286136; Email address: wang_fp@sohu.com (Fengping WANG)

DOI: $10.1007 / \mathrm{s} 40195-013-0226-5$ istry strategy, wet/dry etching and electrochemical approach, etc. ${ }^{[8,9]}$. However, some of them are subjected to certain limitations, such as severe condition, tedious fabrication and presence of organic additives or surfactants. Thus fabrication of metallic nanostructures is still a great challenge.

On the other hand, electrochemical deposition has been demonstrated to be a feasible alternative strategy for fabrication of metals in micro or nanoscale ${ }^{[10,11]}$. Several electrochemical methods were also employed in the preparation of dendrites ${ }^{[12-14]}$. However, the literatures about the fabrication of copper dendrites using ethanol as solution dispersant in electrolyte solution to directly synthesize copper dendrites are rare.

Herein, we report a facile way to fabricate copper dendrites electrodeposition method from acidic copper sulfate solution containing ethanol on the copper electrode directly without template and surfactant. This is the first time that $\mathrm{Cu}$ dendrites are synthesized by such a simple electrodeposition method. The wettability of as-prepared copper dendrites films changed from superhydrophilic to superhydrophobic when ethanol-palmitic acid was added into acidic cop- 
per sulfate solution. In addition, the corrosion resistance of superhydrophobic copper films was also investigated by polarization curves and weight loss measurements.

\section{Experimental}

\subsection{Synthesis of copper dendrites}

The dimension of the $\mathrm{Cu}$ sample $(99.95 \%)$ used in this study is $15 \mathrm{~mm} \times 10 \mathrm{~mm} \times 20 \mathrm{~mm}$. Firstly, the samples were ground down to 2000 grid silicon carbide paper and then polished by diamond paste. Secondly, $\mathrm{Cu}$ samples were cleaned by acetone, alcohol and deionized water to remove the oil from the surface. Thirdly, the samples were immersed in 10 vol. $\% \mathrm{H}_{2} \mathrm{SO}_{4}$ aqueous solution at room temperature for $3 \mathrm{~min}$ to remove the oxidants from the surface, and subsequently cleaned with deionized water. In a typical synthesis of the copper dendrites, $20 \mathrm{~mL}$ of $1 \mathrm{~mol} / \mathrm{L} \mathrm{CuSO}_{4}$ aqueous solution was mixed with $20 \mathrm{~mL}$ of 10 vol. $\% \mathrm{H}_{2} \mathrm{SO}_{4}$ solution at room temperature. Then $100 \mathrm{~mL}$ of absolute ethanol was immediately added to the solution under rapid stirring until a uniform electrolyte solution was obtained at $\mathrm{pH}$ value of 1.5. The electrochemical deposition of the copper dendrites was carried out in a two-electrode cell from the above electrolyte bath at room temperature. The copper sample was mounted as the cathode, and copper plate being the anode. The distance between the two electrodes is about $20 \mathrm{~mm}$. Electrodeposition was carried out with a MPS 3005L-1 dc voltagestabilized power supply, under stirred condition. A direct-current density of $0.015 \mathrm{~A} \cdot \mathrm{cm}^{-2}$ was applied for $240 \mathrm{~s}$. Copper dendrites were deposited on the copper cathodic surface. Finally, the copper cathode was removed from electrolyte solution and rinsed with deionized water, and dried in air.

\subsection{Fabrication of superhydrophobic surfaces}

$100 \mathrm{~mL}$ of $0.1 \mathrm{~mol} / \mathrm{L}$ ethanol-palmitic acid was mixed with $20 \mathrm{~mL}$ of $10 \mathrm{vol} . \% \mathrm{H}_{2} \mathrm{SO}_{4}$ solution at room temperature. Then $20 \mathrm{~mL} 1 \mathrm{~mol} / \mathrm{L} \mathrm{CuSO}_{4}$ solution was quickly added to the solution under rapid stirring until a uniform electrolyte solution was obtained at $\mathrm{pH}$ value of 1.5 . The color of the solution changed immediately to white-blue upon adding copper sulfate solution. Similar experimental procedure was applied to fabricate $\mathrm{Cu}$ dendrites with superhydrophobic property. A direct-current density $0.015 \mathrm{~A} \cdot \mathrm{cm}^{-2}$ was applied for $240 \mathrm{~s}$. Finally, the $\mathrm{Cu}$ cathode was removed from electrolyte solution and rinsed with deionized water, and dried in air.

\subsection{Characterization}

The surface morphology was characterized by scanning electron microscopy (SEM), and Energy Dis- persive X-ray Spectrometer (EDS) was to detect qualitative chemical elements. The composition and crystalline structure of the as-prepared films were identified by powder X-ray diffraction(XRD). The contact angles (CAs) were measured by a JC2000C Digi-drop system under ambient conditions. The average CA value was obtained by measuring five different positions. Electrochemical measurements were performed with a CHI600C electrochemical analyzer system with a platinum plate as the counter electrode and a saturate $\mathrm{Ag} / \mathrm{AgCl}$ electrode as reference electrode. In order to evaluate the corrosion resistant of the samples, the polarization curves measurements and weight loss tests were performed in deionized water at room temperature.

\section{Results and Discussion}

Fig. 1 shows the morphology of the copper dendrites fabricated directly on $\mathrm{Cu}$ substrate. It is observed that the copper dendrites possess remarkable hierarchical structure assembled with some branchlike structures (Fig. 1(a)). At high-magnification those copper dendrites consist of some long main backbone with secondary branches decorated fully by small leaves. The diameter of the backbone is around 3$5 \mu \mathrm{m}$, and its length is up to tens of micrometers. The angle between the branches is almost the same with the value of about $90^{\circ}$, indicating that the copper dendritic crystals grow along a preferential direction.

To further investigating the structure of the copper dendrites, XRD characterization was conducted with the results shown in Fig. 2(a). The three primary diffraction peaks were indexed as the (111), (200), and (220) planes of fcc(face-centered cubic) $\mathrm{Cu}$ $(a=0.306 \mathrm{~nm}$, JCPDS No. 04-0836). The absence of other peaks indicates that those copper dendrites were made of high purity copper. The intensity ratio of (111) to (200) diffraction peaks is 2.3, indicating that the deposited branchlike microstructure intends to grow with the surfaces dominated by the lowest energy (111) facets, and the (111) plane was the primary crystalline plane which might be attributed to the dendrites. The XRD results are consistent with the orientation and geometry of the as-deposited copper dendrites possessing abundant (111) facets. In addition, Fig. 2(b) displays the EDS mapping analysis corresponding to Fig. 1(a). The EDS analysis in a selected domain of the as-prepared sample further testified the $\mathrm{Cu}$ existence, in which the peak of the corresponding elemental $\mathrm{Cu}$ was distinct and no impurities can be detected. Element mass analysis shows that the content of copper is 100 wt.\% (Fig. 2(b)), which is consistent with XRD results. It turned out that copper dendrites could be obtained without impurities using electrochemical deposition approach.

To investigate the evolution of $\mathrm{Cu}$ from copper particles to continuous $\mathrm{Cu}$ film, $\mathrm{Cu}$ samples were deposited at the same current density and different du- 


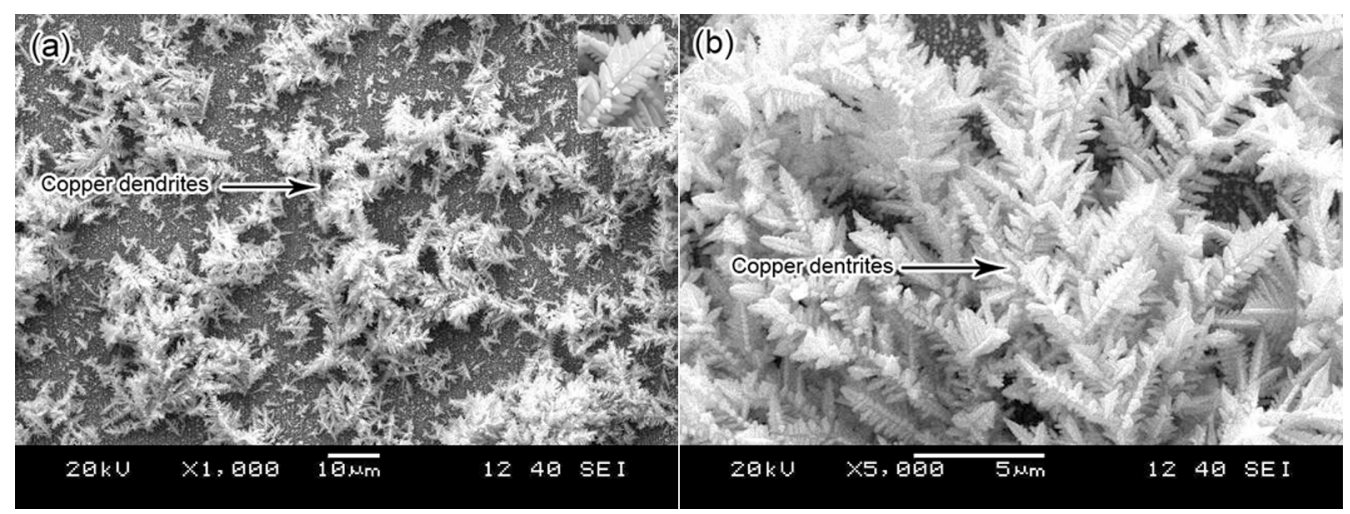

Fig. 1 Low (a) and higher (b) magnified typical SEM images of branchlike copper clusters on copper substrate were prepared by electrodeposition with $0.015 \mathrm{~A} \cdot \mathrm{cm}^{-2}$ current density for $240 \mathrm{~s}$
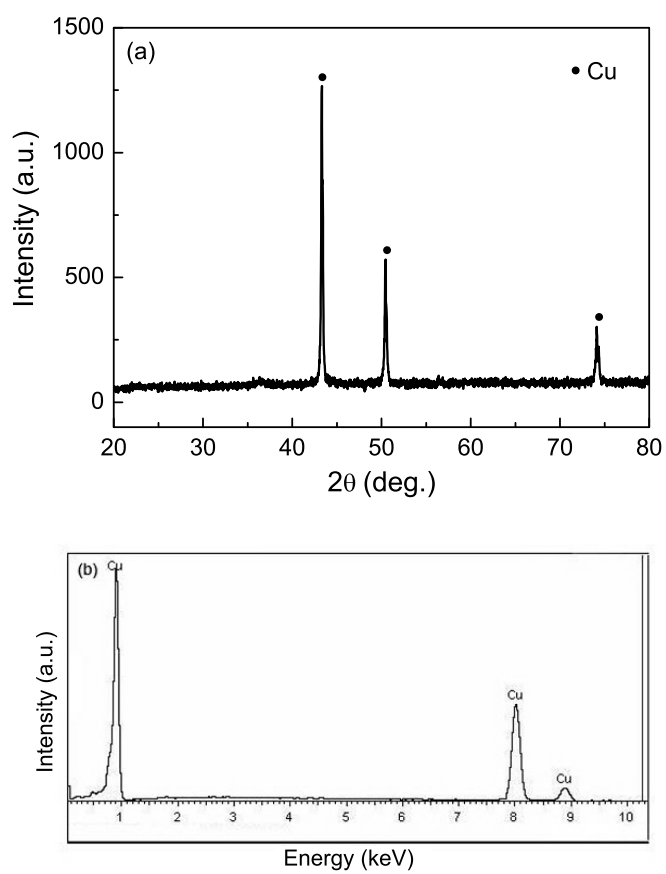

Fig. 2 XRD pattern (a) and the EDS spectrum (b) of the as-deposited copper dendrite as shown in Fig. 1

rations. Fig. 3(a) and (c) show typical SEM images of as-deposited $\mathrm{Cu}$ at the current density of $0.018 \mathrm{~A} \cdot \mathrm{cm}^{-2}$ for $30 \mathrm{~s}$ and $240 \mathrm{~s}$, respectively. Fig. 3(b) and (d) are the corresponding highresolution SEM images. During the electrochemical deposition, $\mathrm{Cu}$ anode and $\mathrm{CuSO}_{4}$ in the electrolyte provided $\mathrm{Cu}^{2+}$ for the deposition on the cathode. For the short deposition time of $30 \mathrm{~s}$, no dendrites were found on the $\mathrm{Cu}$ substrate surface, while only some crystal nucleus could be seen. With increasing deposition duration, this area-selected electrodeposition would become more remarkable, which finally results in the formation of individual copper dendrite, as shown in Fig. 3.

A comparison of Fig. 3(c) with Fig. 1(a) indicates that the rate of nucleation is low at a lower current density, leading to larger crystallites with extended crystal faces. The amount of nucleus is less when the current density is $0.015 \mathrm{~A} \cdot \mathrm{cm}^{-2}$ than the current density is $0.018 \mathrm{~A} \cdot \mathrm{cm}^{-2}$, and the particles were bigger.

Ethanol was widely used as a solvent in chemistry reaction, but few achievement used ethanol as a solution dispersant. It was attractive to synthesize copper dendrites based on ethanol which is an inexpensive and environmentally-friend reagent. In this work, we only used ethanol as solution dispersant to synthesis copper dendrites from acidic copper sulfate solution. At low ionic concentrations, deposition will be favored at points of high strength, leading to the formation of the dendrites. In the present experiment, ethanol decreased effectively the concentration of the plating solution and also decreased ion species mobility which may lead to the formation of copper dendrites. In addition, another reason for forming copper dendrites may attribute to the high $\mathrm{pH}$ of plating solution, tiny hydrogen bubbles evolved during the $\mathrm{Cu}$ deposition process. It is clear that ethanol was only used as a dispersant here. However, the role of ethanol in the formation and morphology control of copper dendrites is still under further investigation.

As an initial application of copper dendrites, it would be interesting to explore if copper dendrites could be used to fabricate superhydrophobic surface. We studied the wetting property of the as-prepared film by contact angle (CA) measurement. The CA of the copper dendrites films (with Fig. 1) is almost $0^{\circ}$, compared with those of native copper surface (CA is about $\left.60^{\circ}\right)$, the increased hydrophilicity on the rough films can be explained by Wenzel's equation ${ }^{[15]}$, which describes the amplification effect of surface roughness on wettability. To construct a superhydrophobic surface, it is necessary to combine a rough surface morphology and low surface-energy coating ${ }^{[16,17]}$. According to the simple procedure described in the experiment part, if ethanol was replaced by ethanolpalmitic acid, and then added in acidic copper sulfate solution, it is even more miraculous to find that the $\mathrm{Cu}$ surface coated with copper dendrites was also obtained (with Fig. 4(a) and (b)). It was distinctive 


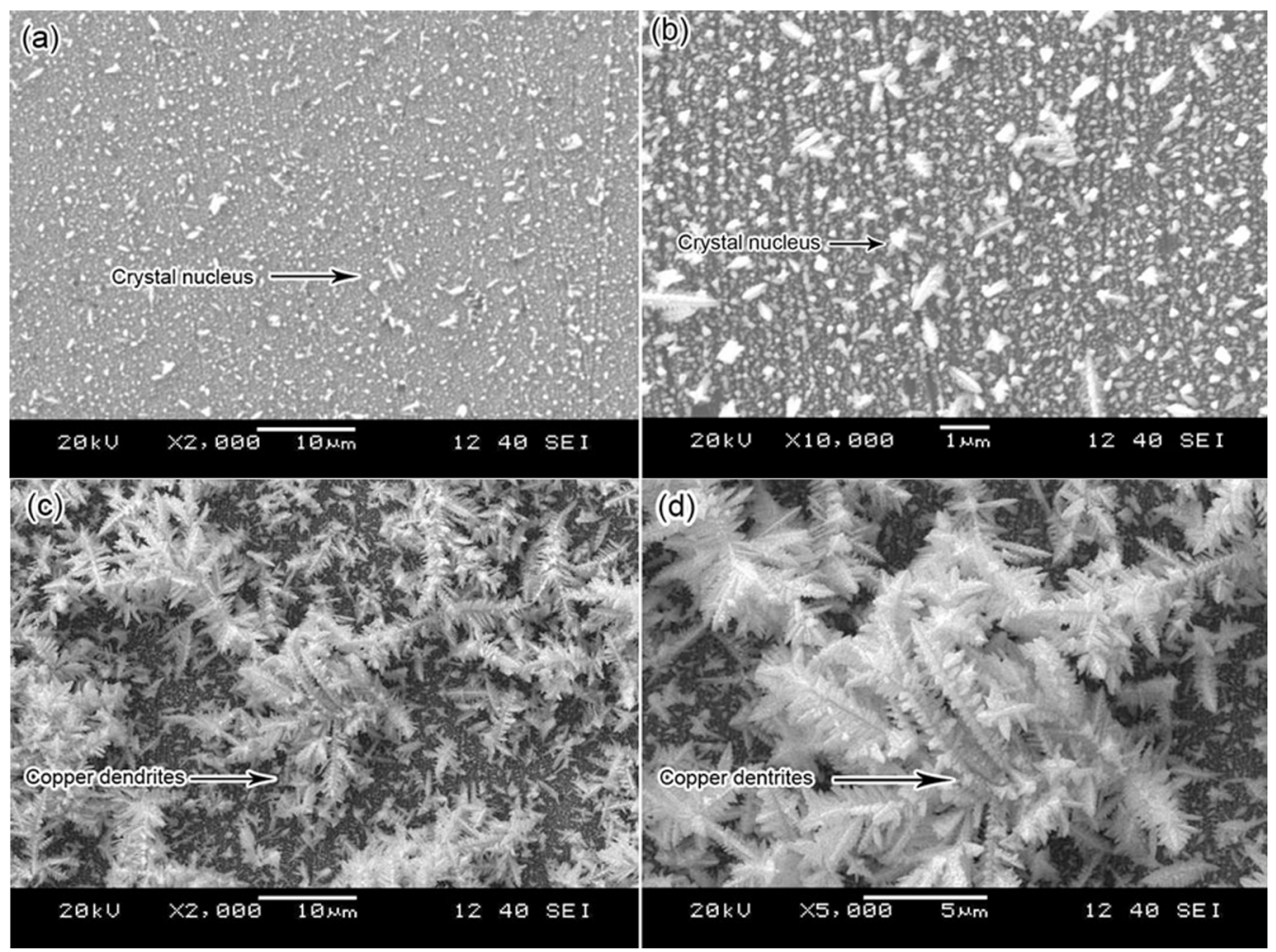

Fig. 3 Typical SEM images of branchlike copper clusters located at $\mathrm{Cu}$ substrate by electrodeposition with 0.018 $\mathrm{A} \cdot \mathrm{cm}^{-2}$ current density for different time: (a, c) $30 \mathrm{~s}$; (b, d) $240 \mathrm{~s}$
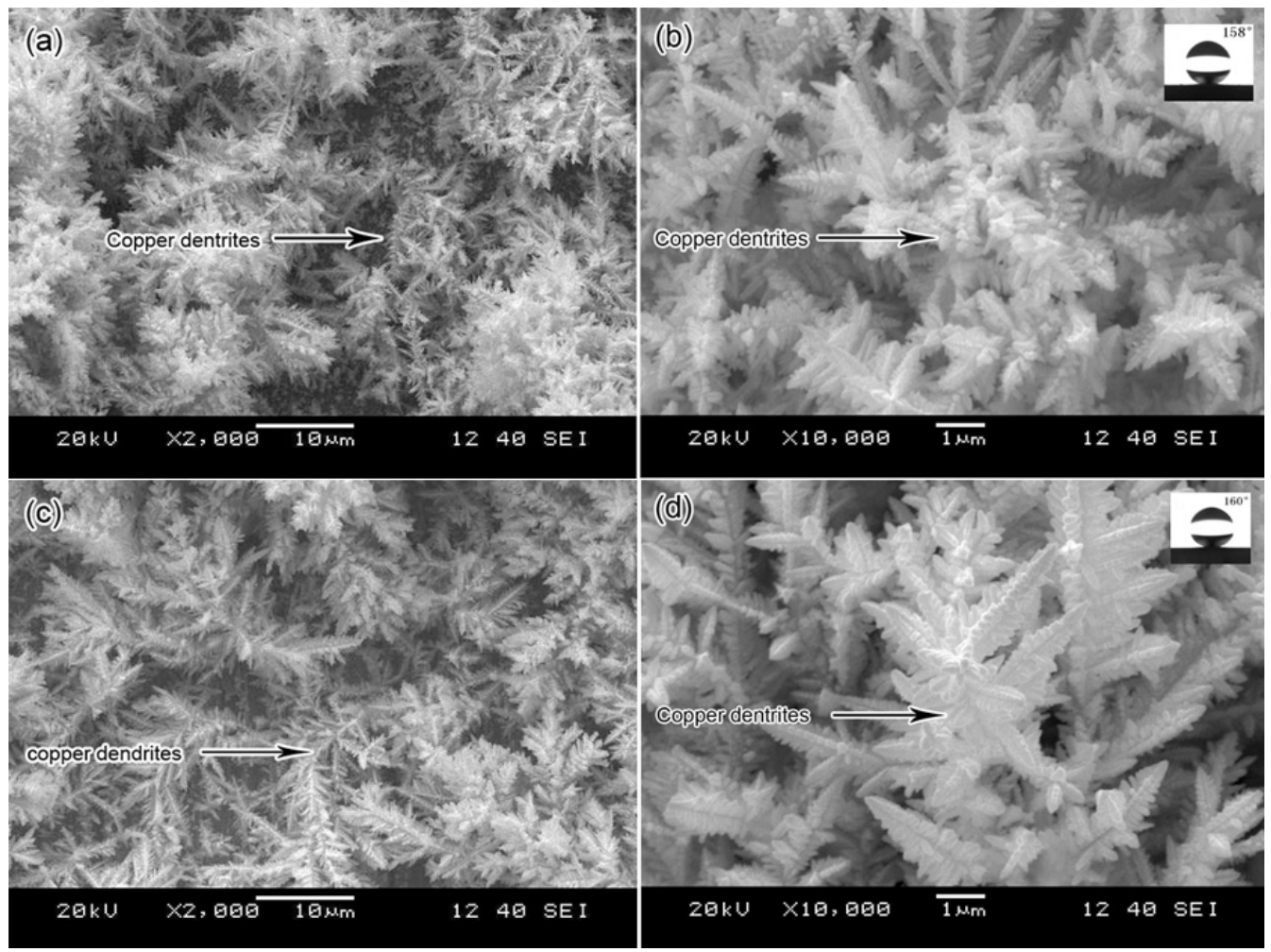

Fig. 4 Typical SEM images of copper dendrites located at $\mathrm{Cu}$ substrate by electrodeposition with $0.015 \mathrm{~A} \cdot \mathrm{cm}^{-2}$ current density for $240 \mathrm{~s}$ in different plating solutions (Insets in Fig. 4(b) and (d) showing the shapes of water droplets): (a, b) ethanol-palmitic acid $+\mathrm{CuSO}_{4}+\mathrm{H}_{2} \mathrm{SO}_{4} ;(\mathrm{c}, \mathrm{d})$ ethanol-myristic acid $+\mathrm{CuSO}_{4}+\mathrm{H}_{2} \mathrm{SO}_{4}$ 


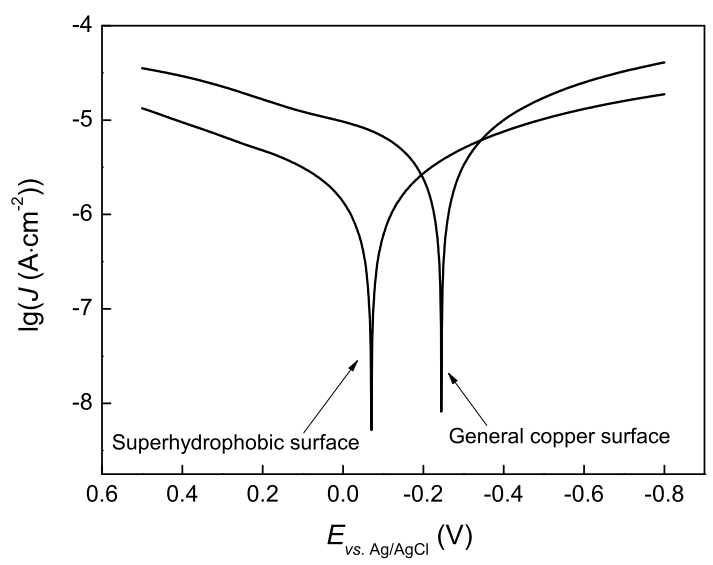

Fig. 5 Tafel polarization curves of general copper surface and superhydrophobic copper surface

that similar copper dendrite structures were obtained compared with the copper dendrites obtained abovementioned (Fig. 1). Those individual copper dendrites grew longer and intercrossed each other. The $\mathrm{CA}$ of the copper dendrites film is $158^{\circ}$. It is clear that the copper dendrites surface modified with palmitic acid shows excellent superhydrophobicity. During CA measurements, a water droplet was hardly able to stick to the surface. The measurement shows that the tilt angle of water-drop is small. If ethanol-myristic acid was added into acidic copper sulfate solution under the same process, it also shows that a similar copper dendrite could be formed in this plating solution (with Fig. 4(c) and (d)). The CA reaches a value of about $160^{\circ}$. Those results show that myristic acid and palmitic acid play the same role in forming the copper dendrite surface with superhydrophobicity. When the rough films are assembled by fatty acids, the lowenergy methyl groups become close-packed on the top surface, which may lead to a low surface free energy and a high CA. The capability of superhydrophobicity is due to the air trapped in the dendrites on the surface. The microclusters and protuberant nanostructures on the top of clusters can effectively prevent the attachment of water droplets and further enhance the hydrophobicity, thus leading to a low sliding angle.

The corrosion resistance of the as-prepared film formed on the copper substrate was investigated by polarization curve measurements. Fig. 5 shows the Tafel polarization curves of the bare $\mathrm{Cu}$ surface and superhydrophobic surface. As clearly seen from Fig. 5, the corrosion potential of superhydrophobic surface $E_{\text {corr }}=-0.07 \mathrm{~V}$, which is positive than that of the bare copper surface $\left(E_{\text {corr }}=-0.18 \mathrm{~V}\right)$. The shift of the $E_{\text {corr }}$ in the positive direction could be linked to the passivation phenomenon of the $\mathrm{Cu}$ surface. The corrosion current density of the $\mathrm{Cu}$ superhydrophobic surface, $j_{\text {corr }}=5.89 \times 10^{-4} \mathrm{~mA} \cdot \mathrm{cm}^{-2}$, which decreased as compared with that of the bare $\mathrm{Cu}$ surface $j_{\text {corr }}=1.78 \times 10^{-3} \mathrm{~mA} \cdot \mathrm{cm}^{-2}$. It reveals that the $\mathrm{Cu}$ with superhydrophobic surface exhibits a better corrosion resistance than the bare one. When the asprepared surface was soaked in water, there was a layer of air on the interface looked like silver mirror between the solid and liquid. It is attributed to the air trapped in the dendrites. Moreover, the superhydrophobic surface soak in water for two week, the air layer still remains.

The corrosion rate of the bare copper, $V_{\text {loss }}$ is $4.4089 \times 10^{-3} \mathrm{~g} \cdot \mathrm{m}^{-2} \cdot \mathrm{h}^{-1}$, according to weight loss measurements, while the corrosion rate of the copper with superhydrophobic surface, $V_{\text {loss }}$ is $1.9137 \times 10^{-3} \mathrm{~g} \cdot \mathrm{m}^{-2} \cdot \mathrm{h}^{-1}$. The weight loss measurements reveal that the corrosion rate of the superhydrophobic surface is much lower than that of the bare one, showing further that the as-prepared surface has anticorrosion performance. The improvement of the corrosion resistance is that the superhydrophobic film protects the $\mathrm{Cu}$ against the oxygen attack in water. Air trapped within the interstices of hierarchical structures greatly increases the air/water interface. In addition, the $\mathrm{Cu}$ samples with the superhydrophobic surface were exposed to air for one month. The CAs still remain the same, indicating the long-term stability of superhydrophobic surfaces.

\section{Conclusion}

A simple, templateless and surfactantless electrochemical technique for fabricating copper dendrites on copper substrate in acidic copper sulfate solution containing ethanol was developed. Ethanol used as solution dispersant plays an important role in the formation of copper dendrites. Based on the roughness caused by the $\mathrm{Cu}$ dendrites, a superhydrophobic surface was fabricated in a similar procedure as long as ethanol-palmitic acid was added into acidic copper sulfate solution. Both Polarization curves and weight loss measurements suggest that the superhydrophobic $\mathrm{Cu}$ films provide enhanced corrosion resistance for the substrate in the aqueous solution.

\section{Acknowledgements}

This work was supported by the Creative Team of the Liaoning Higher Education Institutions of China (No. LT20100055).

\section{REFERENCES}

[1] C. Burda, X.B. Chen, R. Narayanan and M.A. EiSayed, Chem. Rev. 105 (2005) 1025

[2] X.F. Wu, G.Q. Shi, S.B. Wang and P.Y. Wu, Eur. J. Inorg. Chem. 23 (2005) 4775

[3] Y. Chang and H.C. Zeng, Cryst. Growth Des. 4 (2004) 397

[4] C.H. Lu, L.M. Qi, J.H. Yang, D.Y. Zhang, N.Z. Wu and J.M. Ma, J. Phys. Chem. B 108 (2004) 17825

[5] X.L. Xu, J.B. Jia, X.R. Yang and S.J. Dong, Langmuir 26 (2010) 7627

[6] G. Lalwani, A.M. Henslee, B. Farshid, L.J. Lin, F.K. 
Kasper, Y.X. Qin, A.G. Mikos and B. Sitharaman, Biomacrolecules 14 (2013) 900

[7] Q.Z. Hao, B. Wang, J.A. Bossard, B. Kiraly, Y. Zeng, I.K. Chiand, L. Jensen, D.H. Werner and T.J. Huang, J. Phys. Chem. C 116 (2012)7249

[8] Y. Lu, J.L. Song, X. Liu, W.J. Xu, Y.J. Xing and Z.F. Wei, ACS Sustaiable. Chem. Eng. 1 (2013) 102

[9] H. Ogihara, J. Xie, J. Okagaki and T. Saji, Langmuir 28 (2012) 4605

[10] E. Andreoli, D.A. Rooney, W. Redington, R. Gunning and C.B. Breslin, J. Phys. Chem. C 115 (2011) 8725

[11] F.B. Nisancl and U. Demir, Langmuir 28 (2012) 8571
[12] Y.H. Ni, Y.M. Zheng and J.M. Hong, Cryst. Growth Des. 11 (2011) 2142

[13] C.L. Yan and D.F. Xue, Cryst. Growth Des. 8 (2008) 1849

[14] X.J. Zhang, G.F. Wang, X.W. Liu, H.Q. Wu and B. Fang, Cryst. Growth Des. 8 (2008) 1430

[15] D. Jaroslaw and C. Emil, Langmuir 26(2002)18621

[16] L. Feng, S.H. Li, H.J. Li, J. Zhai, Y.L. Song, L. Jiang and D.B. Zhu, Angew. Chem. Int. Edit. 41 (2002) 1221

[17] F. Shi, Y.Y. Song, J. Niu, X.H. Xia, Z.Q. Wang and X. Zhang, Chem. Mater. 18 (2006) 1365 